\title{
The acute effect of a moderate intensity ergocycle exercise on the coagulation parameters in type 2 diabetes mellitus patients: a feasibility study
}

\section{ABSTRACT}

Background. Type 2 diabetes mellitus (T2DM) patients experience higher atherothrombotic risks that could lead to cardiovascular diseases, due to increases in coagulation activities. The role of exercise in altering coagulation activities among T2DM patients is still inconclusive.

This feasibility study aimed to evaluate the immediate effect of a moderate intensity ergocycle exercise, primary on the coagulation parameters and secondary on the systemic inflammation and blood glucose, in otherwise healthy T2DM patients.

Methods. Ten T2DM patients (64 \pm 7 year, $40 \%$ female) performed a 30-minute moderate-intensity ergocycle exercise at $50-60 \%$ of heart rate reserved. Coagulation parameters (activated partial thromboplastin time [aPTT], prothrombin time [PT] and platelet count), erythrocyte sedimentation rate, and blood glucose were assessed before the exercise (TO), immediately after the exercise (T1), and 30 minutes post exercise (T2). One-way repeated measured ANOVA was used to assess the outcomes over time.

Results. All participants completed and adhered to the exercise protocol. There were increases in aPTT and PT but decreases in platelet count at $\mathrm{T} 1$ and $\mathrm{T} 2$ compared to at TO $(P<0.05)$, indicating reduction in the coagula-

Address for correspondence:

Novita Intan Arovah, PhD

Faculty of Sports Science, Yogyakarta State University

Jalan Colombo No 1 Yogyakarta 55281

Phone:+62274513092

e-mail: novita@uny.ac.id

Clinical Diabetology 2020, 9, 4, 219-225

DOI: $10.5603 /$ DK.2020.0029

Received: 02.03.2020

Accepted: 05.05.2020 tion activities. Those values, however, were still within normal ranges. The erythrocyte sedimentation rates were unaffected, while blood glucose decreased from $202 \pm 35 \mathrm{mg} / \mathrm{dL}$ at T0 to $173 \pm 33 \mathrm{mg} / \mathrm{dL}$ and $158 \pm$ $30 \mathrm{mg} / \mathrm{dL}$ at T1 and T2 $(\mathrm{P}<0.01)$.

Conclusion. The 30-minute moderate-intensity ergocycle decreases coagulation activities and blood glucose but does not affect erythrocyte sedimentation rates in T2DM patients. Future studies should focus on the chronic adaptation of the coagulation parameters after ergocycle training among a T2DM patients with coagulation impairments. (Clin Diabetol 2020; 9; 4: 219-225)

Key words: activated partial thromboplastin time, prothrombin time, platelet, erythrocyte sedimentation rate

\section{Introduction}

Type 2 diabetes mellitus (T2DM) patients are at significant risk of developing cardiovascular disease due to the impact of blood glucose elevation on the coagulation system, through a series of actions [1]. First, the increase of blood glucose could induce endothelial abnormalities and stimulate incomplete activation of the coagulation cascade [2]. Second, it causes glycation of proteins responsible for coagulation activities favouring hypercoagulation and stimulates pro-thrombotic states $[3,4]$. Accumulatively, these events trigger the development of atherosclerotic lesions and occlusive thrombus which contribute to the pathogenesis of cardiovascular diseases such as ischemic heart disease and stroke [5]. Thus, hypercoagulation in T2DM patients should be monitored and controlled. 
Among the standard coagulation screening tests for monitoring hypercoagulation conditions in T2DM patients there are the activated partial thromboplastin time (aPTT), prothrombin time (PT) and platelet count performed [6]. The aPTT is used to assess the intrinsic and common coagulation pathway, while the PT assesses the coagulation pathway initiated by tissue factor, as well as the common pathway [7]. A prolonged aPTT and PT is a clinical indicator of fibrinolytic tendency, while a shortened APTT and PT reflects hypercoagulation conditions [8]. Shortened aPTT and PT have been widely reported among T2DM patients [3, 9], along with increases in platelet activities [10]. These indicators, thus, are used for assessing and monitoring hypercoagulation among T2DM patients.

Exercise may play pivotal roles in altering coagulation activities $[11,12]$. Evidence in the literature shows that exercise could increase both blood coagulation and fibrinolysis activities, which each causes opposite effects on the coagulation tendency [11]. The final effect of exercise may depend on the subject characteristics and exercise protocols [13]. In such, the effect of exercise may differ among different age groups or comorbidities and among different exercise intensities, types and duration. Specifically, transient hypercoagulation has been reported after an acute and strenuous exercise in individuals with coronary heart disease symptoms [12] and individuals with type 1 diabetes mellitus [11]. In contrast, increases in fibrinolytic activities were also reported among healthy individuals immediately after vigorous exercise [14], thus offer some protection against the risk of thrombosis and adverse cardiovascular events. These findings, however, may not be readily applied for T2DM patients, since these studies have been conducted in non-T2DM population and were based on vigorous-intensity exercise. Moreover, vigorous type of exercise is not recommended among T2DM patients and that the current physical activity recommendation for T2DM patients calls for a moderate intensity of exercise.

The heterogeneity of findings of the roles of exercise in altering coagulation activities in the literature, thus, substantiate the importance of studying the effect of moderate-intensity exercise on coagulation parameters. The ergocycle-based exercise was selected in this present study because stationary bicycling is one of the exercise types that is recommended for T2DM patients [16]. Thus, the primary aim of this study was to evaluate the feasibility and immediate effect of the moderate-intensity ergocycle exercise on the coagulation parameters, in a view to utilizing the exercise for improving hypercoagulation conditions in
T2DM patients. The secondary aim was to study the immediate effects of the ergocycle exercise on systemic inflammation and blood glucose levels.

\section{Methods}

Participants

Ten otherwise healthy T2DM patients participated in this feasibility study. Patients were recruited from a public hospital in Yogyakarta Indonesia where they received regular care and diabetes medications from their physicians. They obtained clearance from their physicians to do regular exercise and participated in this study. At least three days prior to the commencement of the study, they were required to refrain from having performed any type of heavy physical activity.

\section{Procedures and ethical clearance}

The procedures used in this study were approved by the Ethics Committee of the Faculty of Medicine of Gadjah Mada University (KE/0565). Written informed consent was obtained from each participant. Participants were required to perform a warm-up exercise routine for ten minutes before the ergocycle exercise sessions. The warm-up exercise comprised flexibility exercises involving upper and lower extremities joints. The participants then were required to exercise on a bicycle ergometer to achieve free pedaling at workload up to two kilopounds ( $\mathrm{kp}$ ) until they established a regular and steady pedaling rate at $50 \mathrm{rpm}$. The workload was adjusted to reach the participants' heart rate at $50-60 \%$ of their heart rate reserved and was maintained at this level throughout the exercise period. The heart rate reserve was calculated by subtracting participants' resting heart rate from their maximum heart rate with a formula of 220 minus age. The exercise intensity was confirmed with the Borg Scale for ratings of perceived exertion. It was expected that participants would rate the scale at 10 to 12 during the exercise period to indicate the moderate intensity of exercise. The use of these methods has been validated and considered as a recommended approach for prescribing and monitoring exercise intensity in diabetic individuals [17].

The data collection was conducted on the exercise day with the support of two trained research assistants, two phlebotomists and one laboratory technician from a private clinical laboratory in Yogyakarta. The research assistants assessed participants' blood pressure, heart rate, and perceptions of exertion. The research assistants also ensured that participants adhered to the exercise protocol. Participants were required to take at least 10 hours overnight fast. The phlebotomists collected blood samples three times; (i) an hour after 
a small standardized breakfast, prior to the ergocycle exercise, (ii) within 5 minutes after the ergocycle exercise completed, and (iii) 30 minutes after the exercise. The blood samples were taken with a clean vein puncture (20-gauge needle) from an antecubital vein under controlled venous stasis of 40 torr for less than 30 seconds. All vein punctures were taken in a reclined position.

Participants did not consume sugary drinks or food intake until the last blood sampling was performed. However, they could consume water or non-sugary drink, ad libitum, during the exercise and recovery period. Participants took their regular antidiabetic medication after the final blood sampling. Physicians were on site throughout the exercise and data collection period to monitor and ensure the safety aspect of the protocol, mainly for anticipating the signs of hypoglycemia that might occur.

\section{Measures}

Blood samples for the coagulation testing were prepared using citrate capillary with $3.2 \%$ sodium citrate and with a 1:10 dilution ratio to the specimen. Blood samples for other assessments were treated with EDTA. The complete methods for assessing the study outcomes and the corresponding normal reference range values are available in Table 1.

In addition to prothrombin time, for each participant, the international normalized ratio (INR) was calculated from the PT values according to standard practice. The data received from the laboratory technician were double-checked for ensuring the accuracy of the data. The range checks for data values were conducted and validated before the data were imported into a statistical program for analysis.

\section{Analysis}

The feasibility aspect was assessed by observing the ability and willingness of the participants in adhering to the exercise and data collection protocol. Repeated measured one-way ANOVA (RM-ANOVA) was carried out to assess the differences of outcome measures over time before (T0), immediately after (T1) and 30 minutes (T3) after ergocycle exercise. The Shapiro Wilk test and the sphericity assumption using the Mauchly's test were assessed. The Greenhouse-Geiser correction was used to interpret the within-subject effect for variables that did not meet the sphericity assumption. The pairwise comparisons using the Bonferroni post hoc test were explored if the within-subject effects were significant. Data were analysed using SPSS ${ }^{\circledR}$ v21.0 (SPSS Inc., Chicago, IL, US). A P-value $<0.05$ represented statistical significance.
Table 1. Study outcomes, instruments and normal reference range

\begin{tabular}{|c|c|c|}
\hline $\begin{array}{l}\text { Study } \\
\text { outcomes }\end{array}$ & $\begin{array}{l}\text { Methods/ } \\
\text { /Formula }\end{array}$ & $\begin{array}{l}\text { Normal } \\
\text { reference range }\end{array}$ \\
\hline \multicolumn{3}{|c|}{ Coagulation parameters } \\
\hline $\begin{array}{l}\text { Activated partial } \\
\text { thromboplastin } \\
\text { time }\end{array}$ & $\begin{array}{l}\text { Citrate plasma analysis } \\
\text { with tromborel }\end{array}$ & $23.0-30.2$ second \\
\hline $\begin{array}{l}\text { Prothrombin } \\
\text { time PT }\end{array}$ & $\begin{array}{l}\text { Citrate plasma analysis } \\
\text { with actin and } \mathrm{CaCl}_{2}\end{array}$ & 10.1-11.9 second \\
\hline Platelets & $\begin{array}{l}\text { Hydrodynamic } \\
\text { focussing }\end{array}$ & $\begin{array}{l}150-440 \\
\text { thousand/uL }\end{array}$ \\
\hline \multicolumn{3}{|c|}{ Systemic inflammation } \\
\hline $\begin{array}{l}\text { Erythrocytes } \\
\text { sedimentation } \\
\text { rate }\end{array}$ & $\begin{array}{l}\text { Infrared sensor meth- } \\
\text { od with Westergreen } \\
\text { value presentation }\end{array}$ & $\begin{array}{l}\text { Male: } 0-10 \mathrm{~mm} / \mathrm{hr} \\
\text { Female: } 0-20 \mathrm{~mm} / \mathrm{hr}\end{array}$ \\
\hline Blood glucose & $\begin{array}{l}\text { Spectrophotometry } \\
\text { based on hexokinase } \\
\text { method }\end{array}$ & $<110 \mathrm{mg} / \mathrm{dL}$ \\
\hline
\end{tabular}

PT — prothrombin time

\section{Results}

\section{Participants characteristics}

Participants' mean age was $64 \pm 6.8$, ranged from 47 to 74 years old. Forty percent of participants were female, Fifty percent had education level up to secondary education while the rest had tertiary education. Sixty percent were unemployed or retired and 80 percent were married. None was ex-smoker or smoker. Seventy percent had been diagnosed as having T2DM for more than 5 years. Ninety percent received oral medication and only one participant received insulin therapy. The mean of blood glucose prior to the exercise and an hour after the standardized breakfast was $210 \pm 130$ $\mathrm{mg} / \mathrm{dL}$. Most of them were also hypertensive with the mean of systole and diastole readings of $160 \pm 20$ and $85 \pm 15 \mathrm{~mm} \mathrm{Hg}$, respectively. Lastly, most of them were considered overweight with the average body mass index of $26 \pm 4 \mathrm{~kg} / \mathrm{m}^{2}$.

\section{The feasibility of exercise and data collection protocol}

All participants completed and adhered to the ergocycle exercise procedure and the three data collection. Participants were able to maintain the exercise intensity at moderate levels based on the prescribed intensity which was assessed with \% heart rate reserve and rating of perceived exertion throughout the exercise period. No incident and sign of hypoglycemia were reported by participants and the physician. There was no missing data for all outcome measures at the three time-point. 


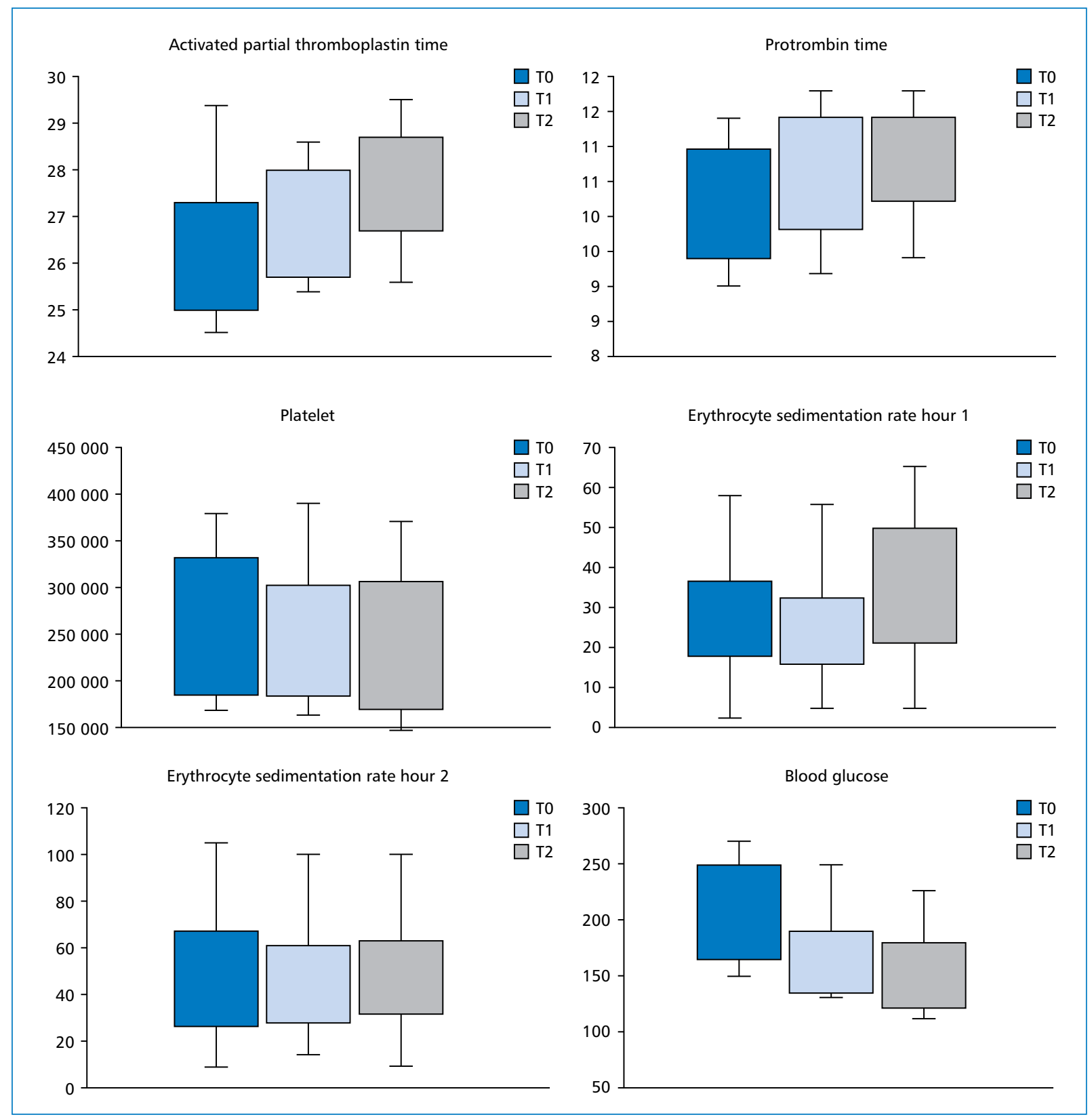

Figure 1. Ranges, interquartile, median and mean of the outcome measures before, immediately after and 30-minute post ergocycle exercise

\section{Outcome measures at baseline, immediately} after and $\mathbf{3 0}$ minutes after ergocycle exercise

Figure 1 illustrates the ranges, interquartile, median and mean, while Table 2 summarizes the means and standard errors of the outcome measures before, immediately after and 30-minute post ergocycle exercise, along with the results from the RM-ANOVA and post hoc analysis.

As shown in Table 2, all coagulant parameters increased immediately after the exercise. The values continued to increase during the recovery period except for platelet count which was the lowest at that period.
Overall, these findings indicated a reduction in coagulation activity. The erythrocytes sedimentation rates were unchanged before and after exercise, while the blood glucose steadily decreased immediately and 30 minutes after exercise from the baseline value $(<0.01)$.

\section{Discussion}

This study assessed the feasibility of the application of a moderate intensity ergocycle exercise, among middle-aged and older T2DM patients to ensure the safety aspect of the exercise and study protocol. Our study demonstrated that the ergocycle exercise and 
Table 2. The outcome measures before, immediately after and 30-minute post ergocycle exercise

\begin{tabular}{|c|c|c|c|c|c|}
\hline & TO & T1 & $\mathrm{T} 2$ & $\mathbf{p}$ & Post hoc \\
\hline \multicolumn{6}{|l|}{ Coagulation parameter } \\
\hline $\begin{array}{l}\text { Activated partial thromboplastin time } \\
\text { (second) }\end{array}$ & $26.4 \pm 0.5$ & $26.8 \pm 0.4$ & $27.7 \pm 0.4$ & $0.01 *$ & $\mathrm{~T} 2>\mathrm{T} 0, \mathrm{~T} 2>\mathrm{T} 1$ \\
\hline Prothrombin time (second) & $10.2 \pm 0.3$ & $10.5 \pm 0.3$ & $10.7 \pm 0.2$ & $0.03^{*}$ & $\mathrm{~T} 2>\mathrm{T} 0$ \\
\hline International normalized ratio & $0.88 \pm 0.02$ & $0.90 \pm 0.02$ & $0.97 \pm 0.03$ & $0.01 *$ & $\mathrm{~T} 2>\mathrm{T} 0$ \\
\hline Platelet [thousand/ml] & $262 \pm 25$ & $252 \pm 24$ & $249 \pm 25$ & $0.02 *$ & $\mathrm{~T} 2<\mathrm{T} 0$ \\
\hline \multicolumn{6}{|l|}{ Systemic inflammation } \\
\hline Erythrocytes sedimentation rate $1[\mathrm{ml} / \mathrm{hr}]$ & $27.0 \pm 11.3$ & $25.4 \pm 9.3$ & $30.4 \pm 11.1$ & $0.07^{\#}$ & - \\
\hline Erythrocytes sedimentation rate 2 [ml/hr] & $47.3 \pm 13.8$ & $49.7 \pm 12.4$ & $50.4 \pm 15.6$ & 0.63 & - \\
\hline Blood glucose [mg/dL] & $202 \pm 35$ & $173 \pm 33$ & $158 \pm 30$ & $<0.01 *$ & $\mathrm{~T} 2<\mathrm{T} 1<\mathrm{T} 0$ \\
\hline
\end{tabular}

\#Greenhouse-Geiser correction; *significant; T0 — baseline; T1 - within 5 minutes after ergocycle exercise; T2 - 30 minutes after ergocycle exercise

study protocol was well-tolerated by middle-aged and older adults with T2DM. To our best knowledge, this present study is the first to report the effect of moderate-intensity ergocycle exercise on clinical coagulation parameters and to explore the association of the coagulation parameters with systemic inflammation and blood glucose among T2DM patients.

The most important clinically relevant findings of this present study were the elevation of aPTT and PT and the reduction of platelet counts that were observed after the short-term moderate-intensity ergocycle exercise, thus, indicated a tendency toward the improvement of coagulation. The findings were in consonance with a study that reported increases in aPTT, PT and fibrinolysis activities after vigorous exercise among diabetes patients [11]. However, the finding was in contrast with the increases in pro-coagulant activity that were reported among individuals with non-T2DM patients after short-term strenuous exercise testing [12]. Similarly, another study also reported the increased risk of thromboembolic incidents in running athletes after a prolonged vigorous exercise session [18]. These conflicting findings support the notion that the effect of exercise on the coagulation system varies and depends upon the exercise type, intensity, duration as well as different subjects' medical conditions [13].

Although there were some variations of findings across previous studies, the absence of the transient hypercoagulation after a short moderate-intensity exercise in this present study was a significant finding because it highlights the safety aspect of the exercise among T2DM patients. The finding, however, needs to be interpreted with caution. The coagulation parameters of this study participants were still within the normal range throughout the study period. Thus, the increases of aPTT and PT as well as the reduction of platelet count in this study, although were statistically significant, may not be clinically significant. Also, the coagulation responses to the exercise on T2DM patients with impaired coagulation system may differ from our study participants with normal coagulation parameters. Future studies, thus, need to further confirm the effect of the exercise on T2DM patients with impaired coagulation parameters.

The increase of erythrocyte sedimentation rate $(E S R)$, one of the systemic inflammation markers, among individuals with T2DM population has been reported [19], thus, the high baseline ESR values in this present study were expected. This present study, however, failed to demonstrate an improvement of ESR after a short-term moderate-intensity exercise. The finding was in contrast with a recent study that reported improvements in ESR in individuals who engaged in regular moderate physical activity levels [20]. Chronic physiological adaptation may be required for ESR improvement. Also, the ESR is a non-specific inflammation marker that could be affected by conditions unrelated to T2DM such as aging, infections or malignancies [20]. Future studies, thus, should consider investigating the effect of both short- and long-term exercise programs on the systemic inflammation and take into account systemic inflammation markers mostly associated with T2DM conditions such as interleukin- 6 and C-reactive protein [21].

Our study also demonstrated steady and significant improvement in blood glucose throughout the study period that was in line with the body of evidence that shows the positive effect of exercise in improving blood glucose levels [22, 23]. It has been recognized that exercise increases blood glucose utilization through the increased activity of the glucose transporter-4, which subsequently promotes the active muscle's glucose uptake to supply the fuel for muscular activity [24]. The exercise also increases cell insulin sensitivity, which 
stimulates blood glucose uptake via a separate channel [24]. These cumulative events eventually reduce the level of circulating blood glucose after the exercise.

Lastly, although the blood glucose improvement in this present study was clinically significant, after 30 minutes of recovery time, the blood glucose was still above the recommended level of $110 \mathrm{mg} / \mathrm{dL}$ [25]. Since further observations were not made after the 30-minute period, a conclusion could not be drawn whether the blood glucose would continue to decrease and eventually achieved the recommended level after the study period. Herein, further studies are recommended to utilize the use of continuous glucose monitoring to confirm the magnitude, trend and duration of blood glucose improvement after exercise interventions are recommended. The information resulted from those studies will be required for assisting the T2DM patients in adjusting their exercise regimen and for guiding clinicians in prescribing anti-diabetic medication to achieve optimal glycemic control while avoiding hypoglycemia.

The key strength of this study was the utilization of the recommended exercise principles for T2DM patients [25]. Thus, findings from this study could be directly applied to T2DM populations. This study has confirmed the feasibility aspect of the moderate-intensity ergocycle exercise. The study also highlighted the safety aspect of the exercise and confirmed its potential role in improving hypercoagulation and blood glucose among middle-aged and adults with T2DM. The findings from this present study, however, must be interpreted in the context of certain limitations. First, this study was intended to assess the feasibility of the exercise protocol and only used a small sample size, thus, it may have resulted in inadequate statistical power for detecting changes and associations in all parameters measured in this study. However, effect sizes could be approximated from this study for calculating the appropriate sample size for future studies. Second, the values of the coagulation parameters were still within the normal range at baseline, thus the findings of this study may not be generally applied for T2DM patients with impaired coagulation. Third, this study only explored the coagulation parameters and did not include the fibrinolytic parameters such as tissue plasminogen activator, plasminogen activator inhibitor-1, and d-dimer. For a complete interpretation of coagulation and fibrinolytic activities, future research is recommended to include these parameters. Last, the ESR that was used in this study is a nonspecific systematic inflammation marker. Thus, the use of systemic inflammation markers more related to T2DM pathogenesis such as CRP and IL-6 is recommended for future research.

\section{Conclusions}

This study has shown that the short-term moderate-intensity ergocycle exercise was well tolerated by middle-aged and older T2DM patients and resulted in improvements in coagulation parameters and blood glucose levels. The systemic inflammation characterized by the erythrocyte sedimentation rates, however, was unaffected. Future studies are recommended to investigate the chronic adaptation of the coagulation parameters after ergocycle training among T2DM patients with coagulation impairments.

\section{Conflicts of interest}

The authors declare to have no conflict of interest.

\section{REFERENCES}

1. Pretorius E. Mechanisms of hypercoagulation and aberrant clot lyses in type 2 diabetes. Mechanisms of Vascular Defects in Diabetes Mellitus. 2017: 377-393, doi: 10.1007/978-3-319-60324-7_17.

2. Lemkes BA, Hermanides J, Devries JH, et al. Hyperglycemia: a prothrombotic factor? J Thromb Haemost. 2010; 8(8): 1663-1669, doi: 10.1111/j.1538-7836.2010.03910.x, indexed in Pubmed: 20492456.

3. Ghosh K. Diabetes as a Prothrombotic State. Mechanisms of Vascular Defects in Diabetes Mellitus. 2017: 361-376, doi: 10.1007/978-3-319-60324-7 16.

4. Carr M. Diabetes mellitus: a hypercoagulable state. Journal of Diabetes and its Complications. 2001; 15(1): 44-54, doi: 10.1016/ s1056-8727(00)00132-x.

5. Loeffen R, Spronk HMH, Ten Cate H. The impact of blood coagulability on atherosclerosis and cardiovascular disease. J Thromb Haemost. 2012; 10(7): 1207-1216, doi: 10.1111/j.15387836.2012.04782.x, indexed in Pubmed: 22578148.

6. Dhule S, Gawali S. Platelet aggregation and clotting time in type 2 diabetic males. National Journal of Physiology, Pharmacy and Pharmacology. 2014; 4(2): 121, doi: 10.5455/ njppp.2014.4.290920131.

7. Mani $\mathrm{H}$. Interpretation of coagulation test results under direct oral anticoagulants. Int J Lab Hematol. 2014; 36(3): 261-268, doi: 10.1111/ijlh.12235, indexed in Pubmed: 24750672.

8. Green D. Interpreting coagulation assays. Blood Coagul Fibrinolysis. 2010; 21 Suppl 1: S3-S6, doi: 10.1097/01. mbc.0000388935.77612.d0, indexed in Pubmed: 20855988

9. Zhao Y, Zhang J, Zhang J, et al. Diabetes mellitus is associated with shortened activated partial thromboplastin time and increased fibrinogen values. PLoS One. 2011; 6(1): e16470, doi: 10.1371/ journal.pone.0016470, indexed in Pubmed: 21297995.

10. Agarwal C, Pujani M, Gahlawat H, et al. Platelet parameters: Can they serve as biomarkers of glycemic control or development of complications in evaluation of type 2 diabetes mellitus? Iraqi Journal of Hematology. 2018; 7(2): 72, doi: 10.4103/ijh.ijh 818.

11. Hilberg T, Eichler E, Gläser D, et al. Blood coagulation and fibrinolysis before and after exhaustive exercise in patients with IDDM. Thromb Haemost. 2003; 90(6): 1065-1073, doi: 10.1160/ TH03-05-0264, indexed in Pubmed: 14652638.

12. Cwikiel J, Seljeflot I, Berge E, et al. Pro-coagulant activity during exercise testing in patients with coronary artery disease. Thromb J. 2017; 15: 3, doi: 10.1186/s12959-016-0127-8, indexed in Pubmed: 28115916.

13. Ribeiro J, Almeida-Dias A, Ascensão A, et al. Hemostatic response to acute physical exercise in healthy adolescents. J Sci Med Sport. 2007; 10(3): 164-169, doi: 10.1016/j.jsams.2006.06.001, indexed in Pubmed: 16844409. 
14. Hilberg T, Gläser D, Reckhart C, et al. Blood coagulation and fibrinolysis after long-duration treadmill exercise controlled by individual anaerobic threshold. Eur J Appl Physiol. 2003; 90(5-6): 639-642, doi: 10.1007/s00421-003-0907-2, indexed in Pubmed: 12883904.

15. Demetz G, Ott I. The Interface between Inflammation and Coagulation in Cardiovascular Disease. Int J Inflam. 2012; 2012: 860301, doi: 10.1155/2012/860301, indexed in Pubmed: 22518344.

16. Sato $Y$, Nagasaki $M$, Kubota $M$, et al. Clinical aspects of physical exercise for diabetes/metabolic syndrome. Diabetes Res Clin Pract. 2007; 77 Suppl 1: S87-S91, doi: 10.1016/j.diabres.2007.01.039, indexed in Pubmed: 17498834.

17. Colberg SR, Swain DP, Vinik Al. Use of heart rate reserve and rating of perceived exertion to prescribe exercise intensity in diabetic autonomic neuropathy. Diabetes Care. 2003; 26(4): 986-990, doi: 10.2337/diacare.26.4.986, indexed in Pubmed: 12663561.

18. Hanke A, Staib A, Görlinger K, et al. Whole blood coagulation and platelet activation in the athlete: a comparison of marathon, triathlon and long distance cycling. Eur J Med Res. 2010; 15(2): 59, doi: doi: 10.1186/cc6430, indexed in Pubmed: indexed in Pubmed: 20452885.

19. Arovah N, Kushartanti B. Moderate-Vigorous Physical Activity and Clinical Parameters in Adults with Type 2 Diabetes Mellitus: A Report from the Walking with Diabetes Study. Romanian Journal of Diabetes Nutrition and Metabolic Diseases. 2019; 26(2): 107-117, doi: 10.2478/rjdnmd-2019-0012.
20. Alende-Castro V, Alonso-Sampedro M, Vazquez-Temprano N, et al. Factors influencing erythrocyte sedimentation rate in adults: New evidence for an old test. Medicine (Baltimore). 2019; 98(34): e16816, doi: 10.1097/MD.0000000000016816, indexed in Pubmed: 31441853.

21. Wang X, Bao W, Liu J, et al. Inflammatory markers and risk of type 2 diabetes: a systematic review and meta-analysis. Diabetes Care. 2013; 36(1): 166-175, doi: 10.2337/dc12-0702, indexed in Pubmed: 23264288.

22. Colberg SR, Sigal RJ, Yardley JE, et al. Physical activity/exercise and diabetes: a position statement of the American Diabetes Association. Diabetes Care. 2016; 39(11): 2065-2079, doi: 10.2337/dc12-0702, indexed in Pubmed: indexed in Pubmed: 27926890.

23. Snowling NJ, Hopkins WG. Effects of different modes of exercise training on glucose control and risk factors for complications in type 2 diabetic patients: a meta-analysis. Diabetes Care. 2006; 29(11): 2518-2527, doi: 10.2337/dc16-1728, indexed in Pubmed: indexed in Pubmed: 17065697.

24. Richter EA, Hargreaves M. Exercise, GLUT4, and skeletal muscle glucose uptake. Physiol Rev. 2013; 93(3): 993-1017, doi: 10.1152/ physrev.00038.2012, indexed in Pubmed: 23899560.

25. American Diabetes Association. 2. Classification and Diagnosis of Diabetes. Diabetes Care. 2017; 40(Suppl 1): S11-S24, doi: 10.2337/dc17-S005, indexed in Pubmed: 27979889 